\title{
Thermal pretreatment promotes the protective effect of HSP70 against tendon adhesion in tendon healing by increasing HSP70 expression
}

\author{
XIAO-MING TANG* ${ }^{*}$ JIAN DAI ${ }^{*}$ and HAI-LANG SUN \\ Department of Orthopedics, The Affiliated Huai'an No. 1 People's Hospital of Nanjing Medical University, \\ Huai'an, Jiangsu 223300, P.R. China
}

Received August 1, 2018; Accepted April 2, 2019

DOI: $10.3892 / \mathrm{mmr} .2019 .10240$

\begin{abstract}
Tendon adhesion is a substantial challenge for tendon repair. Thermal pretreatment (TP) may decrease inflammation by upregulating heat shock proteins (HSPs). The present study intends to identify the function that TP serves when combined with HSP70 overexpression in tendon healing and adhesion in rats. Sprague-Dawley male rats were used to establish a surgically ablative tendon postoperative suture model, and the positive expression of the HSP70 protein was measured using immunohistochemistry. Changes to the blood vessels and collagenous fiber, in addition to the maximum tensile strength and the tendon sliding distance, were detected under a microscope. Finally, HSP70, tumor growth factor $\beta$ (TGF- $\beta$ ), and insulin-like growth factor 1 (IGF-1) mRNA and protein levels were all determined by employing reverse transcription-quantitative polymerase chain reaction and western blot analysis methods. The positive expression of the HSP70 protein increased following TP. Furthermore, TP reduced the infiltration of inflammatory cells and improved the collagenous arrangement, accompanied by an increased maximum tensile force and tendon gliding distance following surgery. In addition, TP increased the mRNA and protein expression levels of HSP70, TGF- $\beta$ and IGF-1. Altogether, TP increases HSP70 expression, thereby reducing postoperative traumatic inflammation and establishing tendon adhesion and promoting tendon healing. Thus, TP may be a potential strategy for the treatment of tendon adhesion.
\end{abstract}

Correspondence to: Dr Hai-Lang Sun, Department of Orthopedics, The Affiliated Huai'an No. 1 People's Hospital of Nanjing Medical University, 1 Huanghe West Road, Huai'an, Jiangsu 223300, P.R. China E-mail: s_hailang@126.com

*Contributed equally

Key words: thermal pretreatment, heat shock protein 70 , tendon healing, tendon adhesion, tendon injury

\section{Introduction}

Tendon injuries are frequent and contribute to a substantial number of cases of morbidity ( $\leq 64 \%$ in patients with rheumatoid arthritis) in sports and in the workplace (1). When the Achilles tendon experiences an injury, the damaged tissue may cause a severe inflammatory reaction, resulting in a 'secondary injury' to the Achilles tendon $(2,3)$. Furthermore, the repair of flexor tendon injuries is complex due to the adhesion forming between the tendons and the surrounding soft tissue post-surgery (4). Another study also provides evidence that adhesion formation has often resulted in limited functional recovery and decreased the ability of the tendon to move smoothly (5). Tendon adhesion is regarded as one of the main problems involved with tendon repair, for which no ideal treatment is currently available (6). Similar to the majority of other tissue repair mechanisms following injury, tendon repair involves a series of molecular and cellular events affected by the location of the injury site, age, sex, nutrition and genetics (7). Thermal pretreatment (TP) serves as a notable factor in the reduction of the inflammatory response and tendon healing by inducing molecular chaperone expression (8).

Heat shock proteins (HSPs), including molecular chaperones, are highly preserved proteins present in eukaryotic and prokaryotic cells and serve a crucial function in various cellular processes, including protein folding, regulation of signaling pathway, degradation of misfolded proteins and the modulation of immune responses (9). HSPs are capable of maintaining cellular homeostasis whenever the microenvironment changes, modulating cell differentiation and proliferation physiologically and pathologically (10). HSP70, a widespread family of molecular chaperones, modulate the protein quality control in addition to homeostasis, serving as an effective drug target for the treatment of neurodegenerative and hyperproliferative disorders (11). Wounds are a type of stress condition that may induce cellular and biochemical responses given that it may promote a significant increase in HSP70 expression in coelomocytes (12). HSPs exhibit properties that promote cell survival in response to all forms of cellular stress (13). Mushtaq et al (14) revealed that HSP70 is necessary for the regulation of epithelial growth, regeneration or adhesion, promoting corneal epithelial wound healing. 
Therefore, the present study hypothesized that the interaction between HSP70 and TP may affect the healing process in tendon injury. The present study intends to investigate the function of TP along with its association with the upregulation of HSP70 in tendon healing and adhesion in rat models simulating tendon injury.

\section{Materials and methods}

Ethical statement. The animal experiments were conducted in accordance with the Guide for the Care and Use of Laboratory Animals as promulgated by the National Institutes of Health (15). Ethical approval was obtained from the ethics committee of The Affiliated Huai'an No.1 People's Hospital of Nanjing Medical University (Huai'an, China).

Experimental animals and establishment of tendon injury rat model. A total of 60 male Sprague-Dawley (SD) rats (aged 10 weeks and weighing between $350-450 \mathrm{~g}$ ) were obtained from the Hubei Research Center of Laboratory Animals (Hubei, China) and randomly assigned into the following groups: Normal (healthy rats) group, model (rats with tendon injury) group, TP (tendon injury rats treated with TP) group, negative control (NC)-small interfering RNA (siRNA) group (tendon injury rats with an injection of $20 \mu \mathrm{l}$ suspension of NC-siRNA-transfected cells at the site of the injury), HSP70-siRNA group (tendon injury rats treated with a $20 \mu \mathrm{l}$ suspension of HSP70-siRNA-transfected cells at the site of the injury) and a combination group (tendon injury rats treated with TP and a $20 \mu \mathrm{l}$ suspension of HSP70-siRNA-transfected cells at the site of the injury) groups ( $\mathrm{n}=10)$. At 4 and 8 weeks following surgery, 5 rats were assigned to each group. The rats were provided with adaptive feeding at a room temperature of $23-25^{\circ} \mathrm{C}$ with a relative humidity between $45-60 \%$ and a light-dark cycle of 12-12 h for 1 week. All rats were allowed ad libitum access to food and water.

The rats were placed under general anesthesia via an intraperitoneal injection of a $1 \%$ pentobarbital sodium $(45 \mathrm{mg} / \mathrm{kg})$ solution and were maintained stationary in a supine position. To obtain a precise incision on the model rats, their legs were shaved and sterilized using iodophor. A $2-\mathrm{cm}$ longitudinal plantar incision was created in the middle of the left paw of the rat. The skin and subcutaneous tissue were sequentially cut to expose the flexor tendon of the deep flexor tendon, and the proximal end of the tendon bifurcation was transversely sliced at $\sim 3 \mathrm{~mm}$. The incision was sutured with a 5-0 tendon suture using the Kessler method (16) and wrapped with sterile gauze. The rats were then administered a subcutaneous injection of sodium chloride solution $(50 \mathrm{ml} / \mathrm{kg})$ on the nape located on their back immediately following the surgery and returned to their cages. They were again provided water and food $4 \mathrm{~h}$ subsequent to returning to their cages. The TP group was then placed in an incubator at a temperature of $45^{\circ} \mathrm{C}$ and remained in the incubator for 15 min until the rectal temperature of rats (measured with a rectal thermometer) reached $\sim 41.5 \pm 0.5^{\circ} \mathrm{C}$. The normal group was subsequently maintained in an incubator at $37^{\circ} \mathrm{C}$ without any form of TP. All rats were allowed to recover at room temperature for $24 \mathrm{~h}$, and none of the rats exhibited symptoms of lethargy and mortality.
Construction of HSP70-siRNA expression vector. The pSUPER-puro expression system (an original short hairpin RNA design) was used to construct the HSP70-siRNA expression vector. Two specific target sequences of HSP70 (GenBank no. NM_005345) were constructed: HSP70-siRNA sense, 5'-GGACGAGUUUGAGCACAAGTT-3' and antisense, 5'-CUUGUGCUCAAACUCGUCCTT-3'; NC-siRNA sense, 5'-UUCUCCGAACGUGUCACGUTT-3' and antisense, 5'-ACG UGACACGUUCGGAGAATT-3'. Each of the two nucleotides in the target sequence contained 9 hairpin structure sequences. The hairpin structure was targeted at the $B g l$ II and $\mathrm{Sal}$ I sites of pSUPER-puro plasmid, thereby constructing a novel recombinant plasmid. H1 RNA polymerase promoter and puromycin were used to select a clone with stable expression.

Culture and transfection of tendon cells. Aponeurosis was homogenized once it was detached from the tendon, digested with mixed collagenase (100 U/ $/$ l; cat. no. 17101015; Gibco; Thermo Fisher Scientific, Inc., Waltham, MA, USA) and placed in Dulbecco's modified Eagle's medium (DMEM) containing $10 \%$ fetal bovine serum (FBS; Gibco; Thermo Fisher Scientific, Inc.) at $37^{\circ} \mathrm{C}$ in a humidified atmosphere of $5 \% \mathrm{CO}_{2}$ for primary culture, and the medium was changed once every 4-5 days. When the medium covered $90 \%$ of the bottom of the medium, the cells were treated with trypsin (1:3; Gibco; Thermo Fisher Scientific, Inc.) to subculture. The cells were passaged until the 3rd generation, which were used for subsequent experiments. Tendon cells involved during the logarithmic growth phase were then seeded into a 12-well plate at a density of $5 \times 10^{4}$ cells/well and finally incubated for a $20 \mathrm{~h}$ interval at $37^{\circ} \mathrm{C}$. Once the medium was replaced by DMEM without the addition of FBS, the cells were transfected with the constructed plasmids $(4 \mu \mathrm{g})$ using Lipofectamine ${ }^{\circledR} 2000$ reagent (Invitrogen; Thermo Fisher Scientific, Inc.) for $48 \mathrm{~h}$ at $37^{\circ} \mathrm{C}$ according to the manufacturer's protocol. Following the aforementioned procedure, the tendon cells used in the in vitro culture were examined. Next, the fluorescent intensity was observed and photographed under a fluorescence inverted microscope (magnification, x200; CX41-12C02, Olympus Optical Co., Ltd., Tokyo, Japan) following $48 \mathrm{~h}$ transfection. The transfection efficiency was calculated according to the following formula: Transfection efficiency $(\%)=$ number of cells with green fluorescent protein (GFP)/total cell number $\times 100 \%(17,18)$. A total of 5 high-power fields were randomly selected, and the mean values were determined.

Observation of tendon adhesion. Tendon healing at the anastomotic site, grading (6), areas of adhesion in peripheral tissues, tendon gliding and smoothness of the aforementioned anastomotic surface were all observed. At week 4 following the surgery, all surgical incisions healed sufficiently without any inflammatory reactions or infection. No rats succumbed to mortality as a result of the surgery. The rats maintained for 4 weeks were then sacrificed; the remaining rats were fed regularly and sacrificed on the 8th week following surgery. Tendon adhesion and gliding in rats were evaluated using a single-blind method (Table I) (6).

Biomechanical test. Following the single-blind method, the rats were injected intraperitoneally with a pentobarbital 
Table I. Scoring standard for tendon adhesion.

Point Severity of tendon adhesion

$0 \quad$ Without adhesion: The tendons glide freely without injury

1-2 Mild adhesion: Fine filaments adhering to peripheral tissues

3-4 Moderate adhesion: Scattered filaments adhering to peripheral tissues

5-6 Severe adhesion: A large number of filaments adhering to peripheral tissues, and the tendon cannot be separated from peripheral tissues and cannot glide

sodium $(150 \mathrm{mg} / \mathrm{kg})$ solution. Following euthanasia, the second toe was disconnected from the carpometacarpal joint, and the deep toe flexor tendon of the second toe was obtained (cut created along $\sim 3 \mathrm{~cm}$ of the carpometacarpal joint). The tendon was then placed in position in a BA-100T mechanical-standard rubber tension test machine (Zhengbang Testing Equipment Co., Ltd., Dongguan, Guangdong, China; http://www.ybzhan.cn/tp29536), ensuring that the tendon was clamped tightly, and that the tendon healing site was located in the middle area between the clamps. The test began with the loading of 1-N force to maintain the proper tendon tension. The initial length was measured. The tendon was stretched to $0.5 \mathrm{~mm}$ at a speed of $0.5 \mathrm{~mm} / \mathrm{s}$, and the pretreatment was conducted a total of three times. Finally, the tendon was loaded to break at a speed of $1 \mathrm{~cm} / \mathrm{min}$. The maximum tensile force of tendon and the sliding distance of the tendon were recorded.

Paraffin sectioning. Following the biomechanical test, the tendon tissue was subsequently removed and treated with $4 \%$ paraformaldehyde at $25^{\circ} \mathrm{C}$ for $4 \mathrm{~h}$. Subsequent to dehydrating with graded ethanol $(75,80,95$, and $100 \%)$, the tissue was cleared using xylene, wax-immersed and then embedded. The paraffin block was placed on a microtome and coarsely repaired to expose the largest section of the tissue block, which was then finely cut into thin slices $\sim 5 \mu \mathrm{m}$ thick. The sections were then placed in a hot water bath (Slidetec WATER; Labsun $\mathrm{GmbH}$, Karlsruhe, Germany). The creased slices were leveled and then placed on a glass slide. The paraffin-embedded specimens were then dried on a $45^{\circ} \mathrm{C}$ hotplate (Slidetec HEAT; Labsun $\mathrm{GmbH}$ ) for a minimum of $5 \mathrm{~min}$.

Hematoxylin and eosin $(H \& E)$ staining. Subsequent to removal from the copying machine, the slices were oven-dried in a $37^{\circ} \mathrm{C}$ oven for $30 \mathrm{~min}$ and dewaxed using graded ethanol (100 and $80 \%$ respectively) until fully hydrated. The slices were then stained using Harris hematoxylin (Shanghai Yuanye Biotechnology Co., Ltd., Shanghai, China) for $10 \mathrm{~min}$ at $25^{\circ} \mathrm{C}$. Then, the slices were washed under tap water for $1 \mathrm{~min}$, differentiated in $1 \%$ hydrochloric acid-ethanol, and rinsed under running water to restore the blue color for $15 \mathrm{~min}$. The slices were then stained with a $1 \%$ ethanol solution of eosin (Shanghai Yuanye Biotechnology Co., Ltd.) for $3 \mathrm{~min}$ at $25^{\circ} \mathrm{C}$, differentiated in $90 \%$ ethanol for $30 \mathrm{sec}$ and washed with a 95\% ethanol solution for 1 min in addition to with dimethyl carbonate (Shanghai Yuanye Biotechnology, Co., Ltd.) for $1 \mathrm{~min}$. The slices were then washed using dimethylbenzene (Sinopharm Chemical Reagent Co., Ltd., Shanghai, China) three times ( 2 min each time) and sealed using a neutral plasma (cat. no. GT21316; Beijing Huayueyang Biotechnology Co., Ltd., Beijing, China; http://huayueyang. biogo.net/). The morphological structure of the tendons was observed under a light microscope (magnification, x200; CX41-12C02, Olympus Optical Co., Ltd.).

Immunohistochemistry. Subsequent to oven-drying in a $37^{\circ} \mathrm{C}$ oven for $30 \mathrm{~min}$, the slices were dewaxed using dimethylbenzene (Sinopharm Chemical Reagent Co., Ltd.) followed by a dehydration process in graded ethanol $(100,95,80$, and $75 \%$ respectively). Following rehydration, endogenous peroxidase activity was inhibited by incubating with $3 \%$ hydrogen peroxide for $1 \mathrm{~h}$ at $37^{\circ} \mathrm{C}$. Then, the slices were washed with phosphate buffer saline (PBS; Sigma-Aldrich; Merck KGaA, Darmstadt, Germany) three times ( 2 min each time) followed by $10 \mathrm{~min}$ of incubation in a sodium citrate buffer $(\mathrm{pH} \mathrm{6.0,}$ $0.01 \mathrm{~mol} / \mathrm{l}$ ) at $96^{\circ} \mathrm{C}$ for antigen retrieval. Subsequent to washing with PBS, the slices were then treated with a rabbit anti-rat HSP70 antibody [1:100; HSP70(W27)(SC-24); Hebei Bio-High Technology Development Co., Ltd., Shijiazhuang, Hebei, China] and placed in a $4^{\circ} \mathrm{C}$ incubator overnight. Subsequent to washing with PBS thrice (5 min each time), a goat anti-rabbit immunoglobulin G secondary antibody (1:2,000; D111018; Shanghai Sangon Biological Engineering Technology \& Services Co., Ltd., Shanghai, China) conjugated to horseradish peroxidase was also added followed by an incubation period at room temperature for $15 \mathrm{~min}$. The slices were then developed using diaminobenzidine (100 $\mu \mathrm{l}$; Wuhan Boster Biological Technology, Ltd., Wuhan, China) and further incubated at room temperature for an additional 5-10 min. Furthermore, the slides were washed with distilled water to terminate the color and further stained using hematoxylin for $5-10 \mathrm{~min}$ at $25^{\circ} \mathrm{C}$. Then, the slides were sealed using neutral plasma. Positive HSP70 expression was present in small brown yellow granules observed in the nucleus or cytoplasm. Five high-power fields were randomly selected for each slice with 1,000 cells per field of view under a light microscope (magnification, x200; CX41-12C02, Olympus Optical Co., Ltd.). The number of positive cells in the field of view were calculated, and the mean positive expression rate was determined as follows: Mean positive expression rate=number of positive cells/total cells $(\%)$.

Histomorphological observation. Tendon tissues of the rats were obtained from the hind limbs of the model rats the 4 and 8 th week following surgery, placed in $10 \%$ buffered formalin for $24 \mathrm{~h}$ and transferred into an $80 \%$ ethanol gradient. Paraffin-embedded tissues were then cut into 5- $\mu \mathrm{m}$ thick slices and stained using H\&E staining, as described above. Tendon development, filament formation, shape, the number of fibroblasts and collagen fiber arrangements were observed under an electron microscope (magnification, x200; CX41-12C02, Olympus Optical Co., Ltd.). The inflammatory reaction of the specimens from rats from the 4 and 8th week post-surgery was evaluated at this juncture of the experiment. The grading 
Table II. Primer sequences for reverse transcription-quantitative polymerase chain reaction.
Item
Primer sequence

Heat shock Forward, 5'-AAGGTGGAGATCATCGCCAA-3'

protein 70 Reverse, 5'-GCGATCTCCTTCTTCATCTTGGT-3'

GAPDH Forward, 5'-AATTCAACGGCACAGTCAAGGC-3'

Reverse, 5'-GGATGCAGGGATGATGTTCTGG-3'

criteria of the inflammatory reaction were established as follows: Grade 1, without or with few lymphocytes; grade 2, a small amount of lymphocytes; grade 3, infiltration of a small amount of neutrophils and lymphocytes in addition to a giant cell reaction; and grade 4, macrophage and inflammatory reactions with the marked infiltration of neutrophils.

Reverse transcription-quantitative polymerase chain reaction (RT-qPCR). Newly formed tendons $(0.1 \mathrm{~g})$ were obtained from the rats at the 4 and 8th week following surgery in each group. Total RNA was then isolated from the tendon tissues of all groups using the TRIzol reagent kit (Gibco; Thermo Fisher Scientific, Inc.) according to the manufacturer's protocol. RNA purity and concentration were assessed using a NanoDrop 2000c Spectrophotometer (Thermo Fisher Scientific, Inc.). Then, HSP70 mRNA was reversely transcribed using the A3500 RT system (Promega Corporation, Madison, WI, USA) at $42^{\circ} \mathrm{C}$ for $1 \mathrm{~h}$ and at $95^{\circ} \mathrm{C}$ for 5 min according to the manufacturer's protocol. RT-qPCR was conducted with synthesized cDNA using a Fast SYBR Green PCR kit (Applied Biosystems; Thermo Fisher Scientific, Inc.). Based on the sequences provided by GenBank (19), specific RT stem-loop primers and primers for PCR amplification (Table II) were designed using Primer Premier 5.0 (Premier Biosoft International, Palo Alto, CA, USA). RT-qPCR analyses were conducted on an ABI 7500 PCR instrument (Applied Biosystems; Thermo Fisher Scientific, Inc.). For the PCR procedure, predenaturation was performed at a temperature of $94^{\circ} \mathrm{C}$ for $5 \mathrm{~min}$ followed by 40 cycles of denaturation at a temperature of $94^{\circ} \mathrm{C}$ for $30 \mathrm{sec}$, annealing at $58^{\circ} \mathrm{C}$ for $30 \mathrm{sec}$ and extension at $72^{\circ} \mathrm{C}$ for $1 \mathrm{~min}$. The mRNA levels of HSP70 and GAPDH genes in the experimental and control groups were measured and calculated utilizing the $2^{-\Delta \Delta \mathrm{Cq}}$ method (20). The quantification cycle $(\mathrm{Cq})$ at which fluorescence intensity is accumulated at a certain quantity was obtained. The $2^{-\Delta \Delta \mathrm{Cq}}$ method was used to determine the ratio of expression of the target genes between the experimental and control groups. The following formula was employed: $\Delta \Delta \mathrm{C}_{\mathrm{q}}=\Delta \mathrm{C}_{\mathrm{q}}$ experimental group $-\Delta \mathrm{C}_{\mathrm{q} \text { control group }}$, and $\Delta \mathrm{C}_{\mathrm{q}}=\mathrm{C}_{\mathrm{qHSP} 70}-\mathrm{C}_{\mathrm{q} \text { GAPDH}}$.

Western blot analysis. Newly formed tendons $(0.1 \mathrm{~g})$ were obtained from rats at the 4th and 8th week following surgery in each group. Total protein was then harvested from the tendon tissues of all groups using a TRIzol reagent kit (Gibco; Thermo Fisher Scientific, Inc.) according to the manufacturer's protocol. The protein concentration of the tendon cells was then measured using a bicinchoninic acid protein

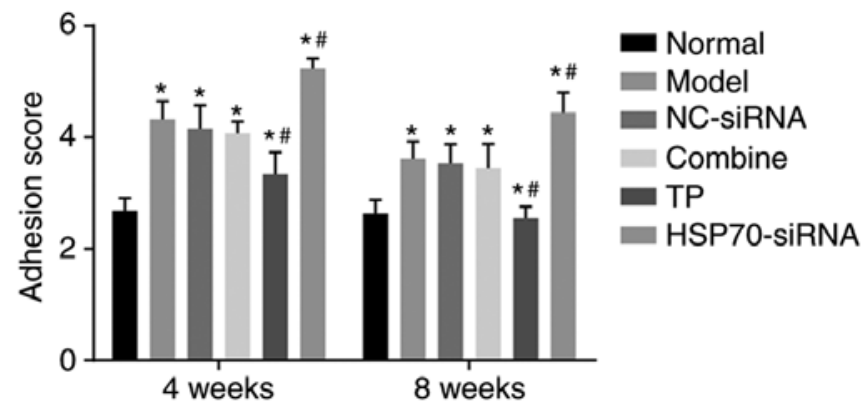

Figure 1. TP reduced tendon adhesion and HSP70-siRNA increased tendon adhesion in rat models with tendon injury. There were 10 rats in each group, with 5 rats observed 4 weeks following surgery and 5 rats observed 8 weeks following surgery. The data were analyzed by repeated-measures analysis of variance. ${ }^{*} \mathrm{P}<0.05$ vs. the normal group; ${ }^{\#} \mathrm{P}<0.05$ vs. the model group. $\mathrm{NC}$, negative control; TP, thermal pretreatment; HSP, heat shock protein; siRNA, small interfering RNA.

assay kit (Beyotime Institute of Biotechnology, Beijing, China). The extracted protein was then mixed with loading buffer, boiled for $10 \mathrm{~min}$ at $100^{\circ} \mathrm{C}$ and loaded at a density of $400 \mu \mathrm{g}$ per well. The proteins were then separated using $10 \%$ sodium dodecyl sulfate-polyacrylamide gel (Wuhan Boster Biological Technology, Ltd.) electrophoresis. The voltage for transfer ranged from 80-120 V. Following the aforementioned transfer, the proteins were transferred onto a polyvinylidene fluoride membrane with wet spinning at a voltage of $100 \mathrm{~V}$ for 45-70 min and subsequently blocked using 10\% dried skimmed milk at room temperature for $1 \mathrm{~h}$. The proteins were then incubated with the following primary antibodies at $4^{\circ} \mathrm{C}$ overnight: Rabbit anti-rat HSP70 monoclonal antibody (1:300; cat no. sc-221731), transforming growth factor- $\beta$ (TGF- $\beta$; 1:200; cat. no. sc-203295), insulin-like growth factor I (IGF-1; 1:200; cat. no. sc-1422) and $\beta$-actin (1:100; cat. no. sc-47778). All antibody used above were purchased from Santa Cruz Biotechnology, Inc. (Dallas, TX, USA). The proteins were washed using tris-buffered saline with $0.5 \%$ Tween 20 (TBST) three times ( 3 min per wash). Then, the secondary antibody, horseradish peroxidase-conjugated goat anti-mouse antibody (1:1,000, ab97057, Abcam, Cambridge, MA, USA) were added and incubated at room temperature for $1 \mathrm{~h}$. Subsequent to washing with TBST for an additional three times (each for $5 \mathrm{~min}$ ), the samples were finally developed using an enhanced chemiluminescence method (Bio-Rad Laboratories, Inc., Hercules, CA, USA). Gray values of the protein bands were analyzed by using SPSS version 21.0 (IBM Corp. Armonk, NY, USA). The ratio of the gray values targeting proteins were determined and $\beta$-actin was used as an internal reference for targeted protein levels.

Statistical analysis. SPSS version 21.0 (IBM Corp., Armonk, NY, USA) was used for statistical analysis. Measurement data were expressed as the mean \pm standard deviation. Comparisons among groups were analyzed utilizing a one-way analysis of variance, followed by Tukey's post hoc test. Repeated measures analysis of variance was used for comparisons among multiple groups at multiple time points. $\mathrm{P}<0.05$ was considered to indicate a statistically significant difference. 


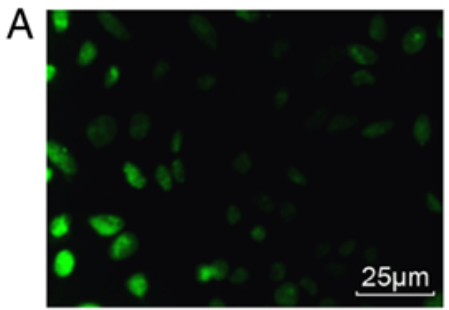

NC-siRNA

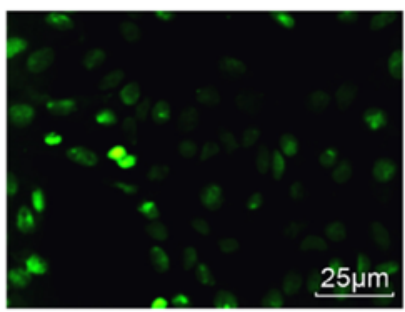

HSP70-siRNA-1

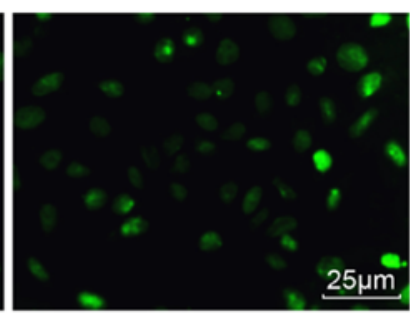

HSP70-siRNA-2

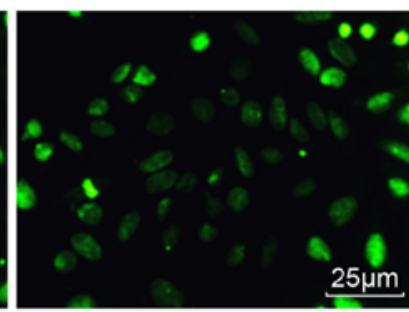

HSP70-siRNA-3
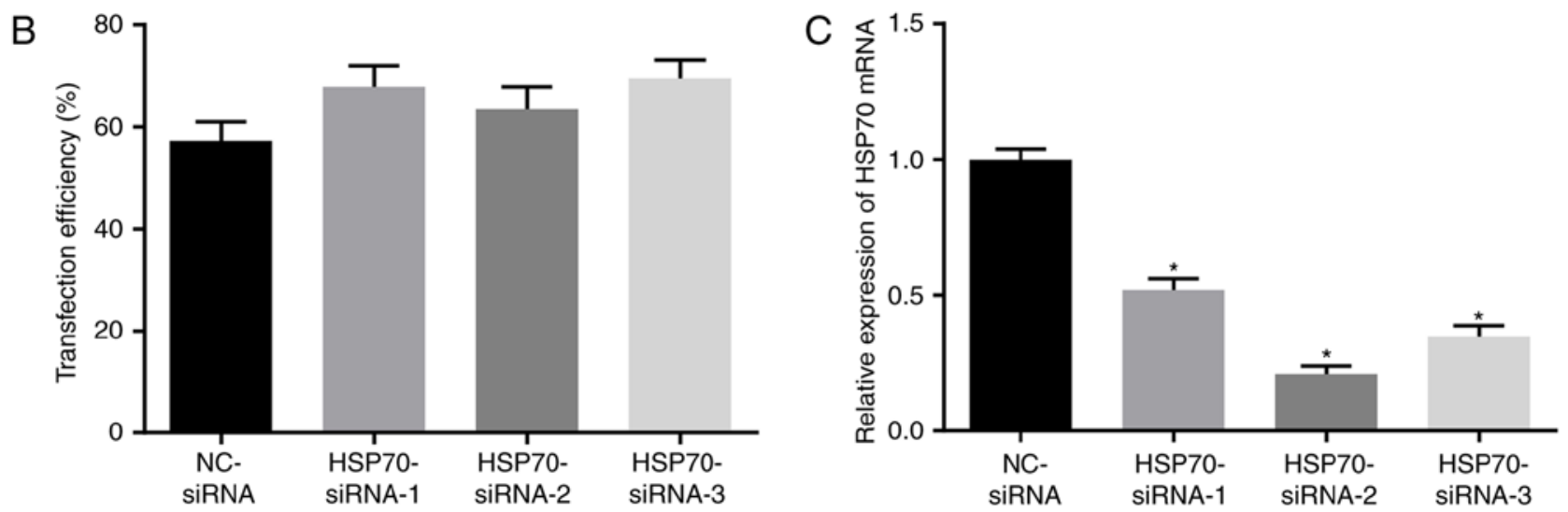

Figure 2. Successful transfection was confirmed based on GFP expression observed under an inverted fluorescence microscope. (A) GFP expression in each group subsequent to transfection observed under a fluorescence microscope (magnification, x400). (B) Transfection efficiency in each group. (C) HSP70 mRNA expression levels in the cells transfected with different siRNAs detected by reverse transcription-quantitative polymerase chain reaction. ${ }^{*} \mathrm{P}<0.05$ vs. the NC-siRNA group. The data were analyzed using one-way analysis of variance. The experiment was conducted three times independently. NC, negative control; HSP, heat shock protein; GFP, green fluorescent protein; siRNA, small interfering RNA.

\section{Results}

TP reduces tendon adhesion and HSP70-siRNA increases tendon adhesion. Tendon adhesion in each group was observed at weeks 4 and 8 subsequent to the operation. A single-blind method was used for the evaluation of the tendon adhesion in each group. As presented in Fig. 1, at the 4th week following surgery, the adhesion grades of tendon adhesion among the model, TP, HSP70-siRNA, NC-siRNA and combined groups exhibited significant differences compared with the normal group $(\mathrm{P}<0.05)$. Compared with the model group, no significant difference was observed between the NC-siRNA group and the combined group $(\mathrm{P}>0.05)$. Compared with the model group, the TP group presented a significantly lower adhesion score $(\mathrm{P}<0.05)$, whereas the HSP70-siRNA group exhibited a significantly increased adhesion score $(\mathrm{P}<0.05)$. These scores indicated that TP reduces tendon adhesion, whereas HSP70 gene silencing increases adhesion.

Successful transfection of HSP70-siRNA in vitro. Five days following transfection, a fluorescence inverted microscope was used to observe GFP in transfected tendon cells. The transfection was confirmed through GFP expression tracked by fluorescence photography. The transfection confirmed an efficiency between 50 and $70 \%$ (Fig. 2A-B), indicating that the transfection of HSP70-siRNA was successful. RT-qPCR was used to detect HSP70 expression upon transfection with different siRNAs (Fig. 2C). Compared with the NC-siRNA group, HSP70 mRNA expression levels in the HSP70 siRNA-1, HSP70 siRNA-2 and HSP70 siRNA-3 groups were significantly decreased $(\mathrm{P}<0.05)$, and the greatest decrease in HSP70 mRNA expression was observed in the HSP70 siRNA-2 group. Therefore, HSP70 siRNA-2 was selected for subsequent experiments.

Morphological changes in the tendon tissues in rats with tendon injuries. A microscope was used to thoroughly examine morphological changes in blood vessels and collagenous fiber. Four weeks post-surgery, the model, NC-siRNA and combined groups exhibited active fibroblast and collagen fiber proliferation, and the infiltration of inflammatory cells and collagen fibers at the broken ends in addition to the anastomosis site, including the production of active phagocytes. Substantial capillary proliferation was observed in the paratendon at the anastomosis site, with notable congestive edema and infiltration of inflammatory cells, which mainly included neutrophils and monocytes. The inflammation at the anastomosis site was categorized into 3 grades. Comparatively, fewer inflammatory cells were observed in the TP group, and the inflammatory response was characterized as grade 2 . The HSP70-siRNA group exhibited a higher number of inflammatory cells in comparison with the normal group. Given this increase, the inflammatory response was characterized as grade 4 . At week 8 following surgery, congestive edema and infiltration of inflammatory cells at the broken ends of the tendon were reduced in the model, NC-siRNA and combined groups. Substantial fibroblast proliferation was observed in the tendon parenchyma close to the broken ends, and newly formed collagen was additionally observed in the broken ends. The number of novel capillaries formed was increased, 

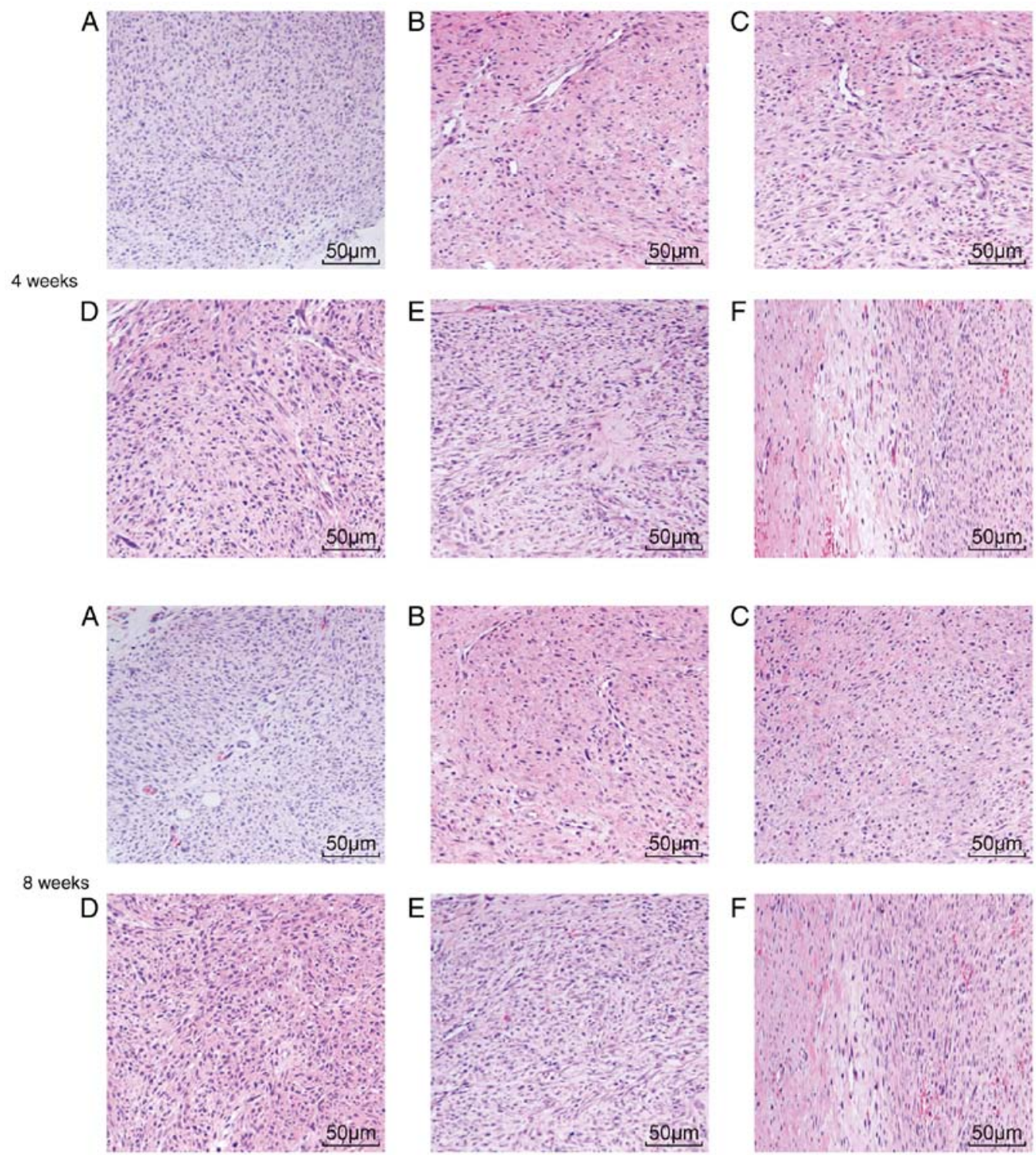

Figure 3. H\&E staining indicated that TP may alleviate the infiltration of inflammatory cells and collagenous arrangement (magnification, $\mathrm{x} 200$ ). (A) H\&E staining in the normal group. (B) H\&E staining in the model group. (C) H\&E staining in the negative control-siRNA group. (D) H\&E staining in the combined group. (E) H\&E staining in the TP group. (F) H\&E staining in the heat shock protein 70-siRNA group. H\&E, hematoxylin and eosin; TP, thermal pretreatment; siRNA, small interfering RNA.

whereas the caliber was broader compared with that noted one week prior. The TP group presented a regular arrangement of the collagen fibers that were almost parallel with the long axis of the tendon. The number of infiltrated inflammatory cells and capillaries at the anastomosis site and paratendon were considerably reduced compared with that noted 4 weeks previously. The inflammatory response was reduced to grade 1. HSP70-siRNA resulted in an irregular arrangement of collagen fibers at the anastomosis site. Net migration of the tendon fibroblasts from the anastomosis site to the outer tendon was observed. Four weeks following surgery, the TP group exhibited a notable difference in the optimized infiltration of inflammatory cells at the early stage and a more regular arrangement of collagen fibers in the middle and late stages compared with the model, NC-siRNA, HSP70-siRNA and combined groups (Table III). These results demonstrated that TP optimized infiltration of inflammatory cells and the collagenous arrangement.
Table III. Inflammatory response grading.

\begin{tabular}{lccc}
\hline & & \multicolumn{2}{c}{ Grade } \\
\cline { 3 - 4 } Group & Sample & 4th week & 8th week \\
\hline Normal & 10 & 0 & 0 \\
Model & 10 & 3 & 2 \\
Negative control-siRNA & 10 & 3 & 2 \\
Combined & 10 & 3 & 2 \\
Thermal pretreatment & 10 & 2 & 1 \\
Heat shock protein & 10 & 4 & 3 \\
70-siRNA & & & \\
\end{tabular}

There were 10 rats in each group, with 5 rats observed 4 weeks following the surgery and 5 rats observed 8 weeks following the surgery. siRNA, small interfering RNA. 
Table IV. Maximum tensile strength and tendon gliding distance 4 and 8 weeks following surgery.

\begin{tabular}{|c|c|c|c|c|c|}
\hline \multirow[b]{2}{*}{ Group } & \multirow[b]{2}{*}{ Sample } & \multicolumn{2}{|c|}{ Maximum tensile strength $(\mathrm{N})$} & \multicolumn{2}{|c|}{ Tendon gliding distance $(\mathrm{mm})$} \\
\hline & & 4th week & 8th week & 4th week & 8th week \\
\hline Normal & 10 & $84.48 \pm 4.34$ & $88.47 \pm 5.41$ & $3.30 \pm 0.34$ & $3.26 \pm 0.45$ \\
\hline Model & 10 & $61.55 \pm 3.41^{\mathrm{a}}$ & $70.67 \pm 4.67^{\mathrm{a}}$ & $1.90 \pm 0.27^{\mathrm{a}}$ & $1.86 \pm 0.22^{\mathrm{a}}$ \\
\hline Negative control-siRNA & 10 & $59.45 \pm 3.56^{\mathrm{a}}$ & $69.58 \pm 4.42^{\mathrm{a}}$ & $1.94 \pm 0.17^{\mathrm{a}}$ & $1.82 \pm 0.32^{\mathrm{a}}$ \\
\hline Combine & 10 & $60.54 \pm 4.21^{\mathrm{a}}$ & $69.77 \pm 3.56^{\mathrm{a}}$ & $1.91 \pm 0.57^{\mathrm{a}}$ & $1.80 \pm 0.38^{\mathrm{a}}$ \\
\hline Thermal pretreatment & 10 & $79.05 \pm 4.68^{\mathrm{a}, \mathrm{b}}$ & $87.34 \pm 4.53^{\mathrm{b}}$ & $2.51 \pm 0.41^{\mathrm{a}, \mathrm{b}}$ & $3.21 \pm 0.24^{\mathrm{b}}$ \\
\hline Heat shock protein 70 -siRNA & 10 & $45.78 \pm 4.32^{\mathrm{a}, \mathrm{b}}$ & $51.67 \pm 3.78^{\mathrm{a}, \mathrm{b}}$ & $1.34 \pm 0.56^{\mathrm{a}, \mathrm{b}}$ & $1.21 \pm 0.58^{\mathrm{a}, \mathrm{b}}$ \\
\hline
\end{tabular}

There were 10 rats in each group, with 5 rats observed 4 weeks following the surgery and 5 rats observed 8 weeks following the surgery. The data were analyzed using repeat measures analysis of variance. ${ }^{\mathrm{a}} \mathrm{P}<0.05$ vs. the normal group; ${ }^{\mathrm{b}} \mathrm{P}<0.05$ vs. the model group. siRNA, small interfering RNA.

TP increases the maximum tensile force and tendon gliding distance in rats with tendon injury. Biomechanical tests were performed to detect the maximum tensile strength along with the tendon sliding distance. As presented in Table IV, at week 4 post-surgery, significant differences were identified in maximum tensile strength and tendon gliding distance in all 5 groups compared with the normal group $(\mathrm{P}<0.05)$. Compared with the model group, the NC-siRNA and combine groups exhibited no differences in maximum tensile strength and tendon gliding distance $(P>0.05)$. In contrast, the TP and HSP70-siRNA groups presented a significantly greater maximum tensile strength and tendon gliding distance compared with the model group $(\mathrm{P}<0.05)$. At week 8 following surgery, no significant differences were noted in the tendon gliding distance between the normal and TP groups $(\mathrm{P}>0.05)$. These results suggested that TP positively increased the tensile strength and tendon sliding distance.

Pathological changes in the tendon tissues in rats with tendon injuries. H\&E staining was used to detect any pathological changes the tendon tissue in SD male rats. At weeks 4 and 8 following surgery, the normal group exhibited intact collagen fibers, orderly arranged tendon cells with clear nuclei of uniform size, evenly distributed chromo-plasm and an intact nuclear membrane without cell swelling. At week 4 subsequent to surgery, irregular collagen fibers were observed at the tendon anastomotic sites in the model, NC-siRNA and combined groups. In addition, few fibroblasts and collagenocytes of irregular shape and inflammatory cell infiltration were observed in the peripheral tissue. Collagen fibers observed in the rat tendons at week 8 following surgery were arranged in a more orderly manner compared with those observed at the 4th week following surgery. Collagen fibers at the tendon anastomotic sites in the TP group at week 8 following surgery were more orderly arranged compared with those at 4th week post-surgery. In addition, collagen fibers and fibroblasts exhibited a regular arrangement. At the 8th week following surgery, considerable scar tissue with pleomorphic fiber formed at the incision site of the rat tendons in the model group, whereas the scar tissues of the TP group were more mature and better aligned, exhibiting lower inflammatory cell infiltration. At week 4 and 8 post-surgery, the HSP70-siRNA group exhibited extremely disordered collagen fibers at the tendon anastomotic sites (Fig. 3A-F). These results indicated that TP may improve inflammatory cell infiltration and collagenous arrangement.

TP increases HSP70-positive expression in the tendon tissues. Immunohistochemistry was used to measure the positive expression of the HSP70 protein. The tendon cells exhibiting yellow-brown particles in either the cytoplasm or nucleus were considered to be HSP70 positive. The immunohistochemical results demonstrated that HSP70 protein expression was particularly low in the normal group and the highest in the TP group. At week 8 following surgery, HSP70 protein expression levels significantly increased in the TP group compared with the normal group $(\mathrm{P}<0.05)$. The normal, model, NC-siRNA and combined groups exhibited various levels of HSP70 expression, but these levels were lower compared with the TP group. HSP70 expression levels in the HSP70-siRNA group were also significantly reduced compared with the normal group ( $\mathrm{P}<0.05$; Fig. 4A-B). Thus, TP increased HSP70 protein expression.

TP increases HSP70 mRNA expression in tendon tissues. RT-qPCR was used to detect HSP70 mRNA levels. As presented in Fig. 5A-B, compared with the control (normal) group, significant differences in HSP70 mRNA expression levels were noted among the 5 groups $(\mathrm{P}<0.05)$. Compared with the control group, HSP70 mRNA levels were significantly increased in the TP group $(\mathrm{P}<0.05)$ but reduced in the HSP70-siRNA group $(\mathrm{P}<0.05)$. Compared with the model group, HSP70 mRNA levels between the NC-siRNA group and the combined group exhibited no significant differences (P>0.05). HSP70 mRNA levels were significantly enhanced in the TP group $(\mathrm{P}<0.05)$ and reduced in the HSP70-siRNA group $(\mathrm{P}<0.05)$ compared with the model group. These results indicated that TP may increase HSP70 mRNA levels.

TP increases HSP70, TGF- $\beta$ and IGF-1 protein levels. Western blot analysis was used to detect HSP70, TGF- $\beta$ and IGF-1 protein levels. As presented in Fig. 6A-D, compared with the 


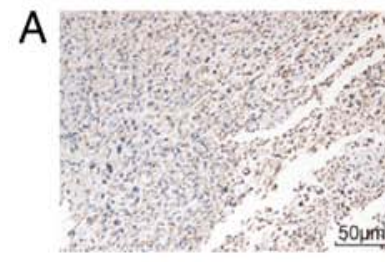

Normal

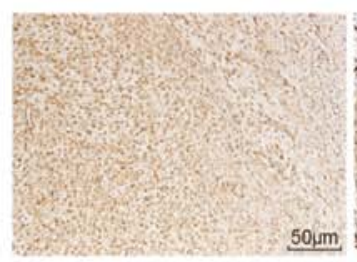

Combine

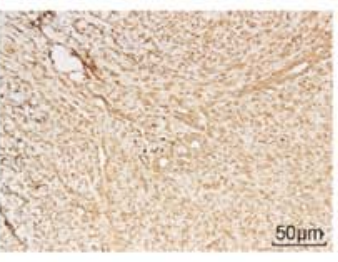

Model

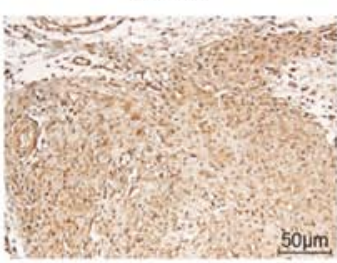

TP

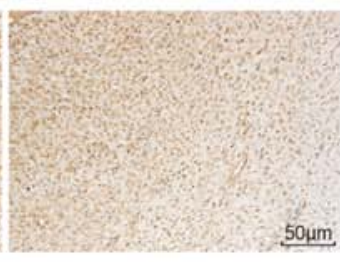

NC-siRNA

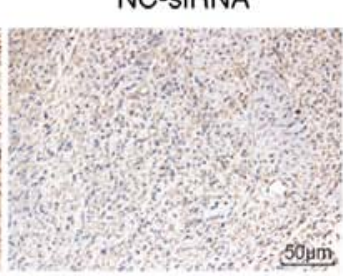

HSP70-siRNA

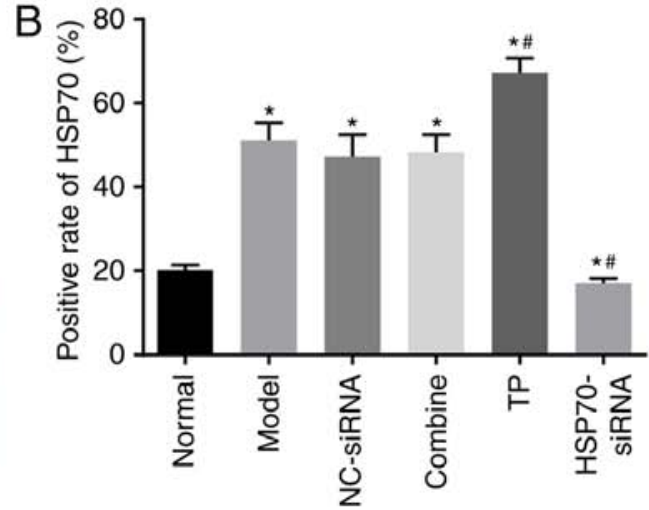

Figure 4. Immunohistochemical analysis revealed increased positive expression of HSP70 protein in rats undergoing TP. There were five rats in each group. (A) Immunohistochemical staining of tendon tissues in each group (magnification, x200); (B) Relative HSP70 expression in the tendon tissue of rats in each group. The data were analyzed using one-way analysis of variance. ${ }^{*} \mathrm{P}<0.05$ vs. the normal group; ${ }^{\prime} \mathrm{P}<0.05$ vs. the model group. NC, negative control; TP, thermal pretreatment; HSP, heat shock protein; siRNA, small interfering RNA.
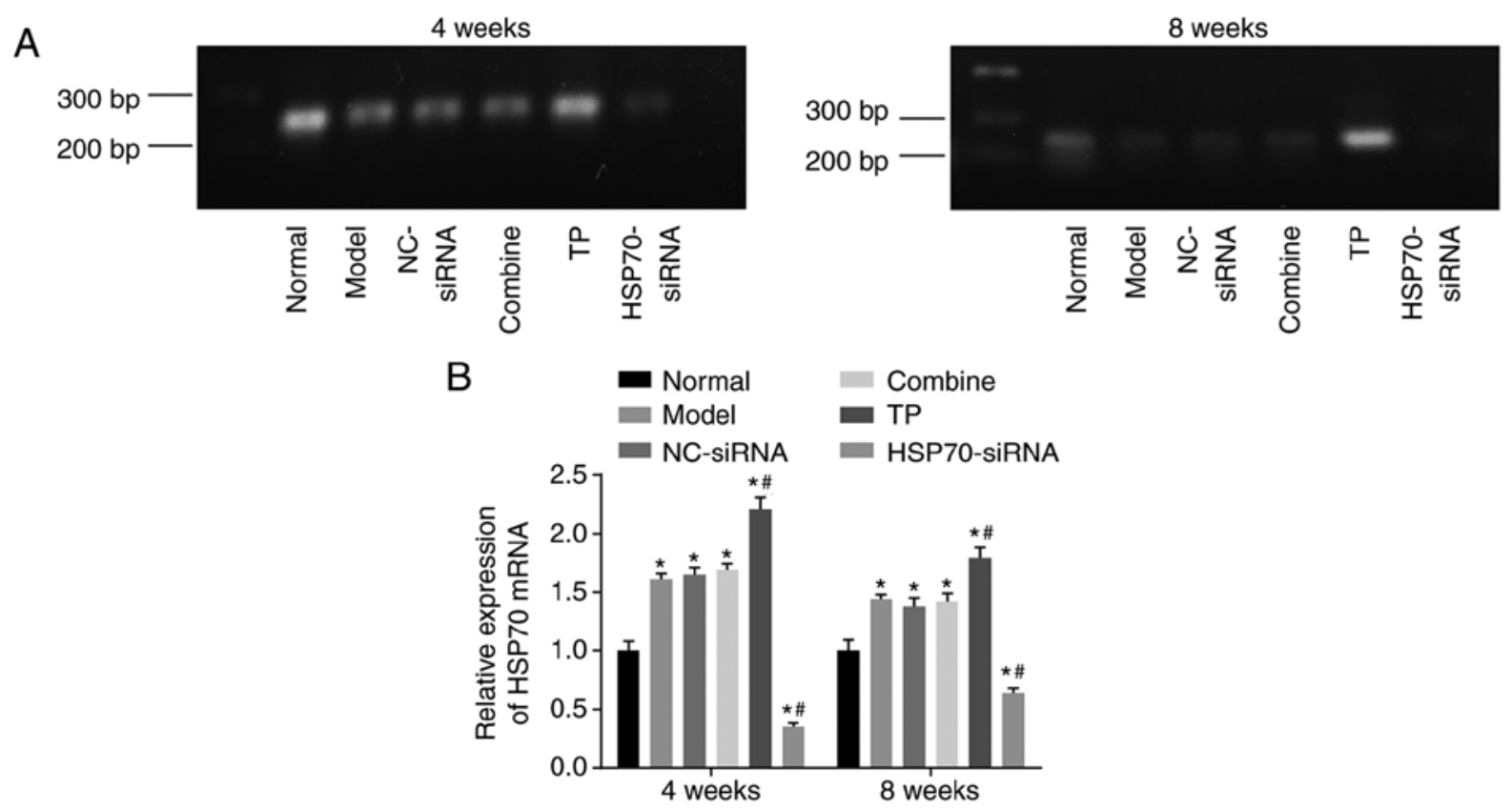

Figure 5. Reverse transcription-quantitative polymerase chain reaction revealed that TP increased HSP70 mRNA expression in rat models with tendon injuries. There were 10 rats in each group, with 5 rats observed 4 weeks following surgery and 5 rats observed 8 weeks following surgery. (A) Bands of HSP70 mRNA expression in each group. (B) Quantification analysis of HSP70 mRNA expression levels in each group. Data were analyzed by repeat measures analysis of variance. "P<0.05 vs. the normal group; ${ }^{\#} \mathrm{P}<0.05$ vs. the model group. $\mathrm{NC}$, negative control; TP, thermal pretreatment; HSP, heat shock protein; siRNA, small interfering RNA; bp, base pairs.

control group, significant differences in HSP70, TGF- $\beta$ and IGF-1 protein levels were noted in the 5 other groups $(\mathrm{P}<0.05)$. Compared with the control group, HSP70 protein expression levels were significantly enhanced in the TP group $(\mathrm{P}<0.05)$ but reduced in the HSP70-siRNA group $(\mathrm{P}<0.05)$. Compared with the model group, HSP70, TGF- $\beta$ and IGF-1 protein levels between the NC-siRNA and combined groups did not differ $(\mathrm{P}>0.05)$, and HSP70, TGF- $\beta$ and IGF-1 protein levels were significantly increased in the TP group $(\mathrm{P}<0.05)$ but decreased in the HSP70-siRNA group $(\mathrm{P}<0.05)$. The aforementioned results demonstrated that TP enhances HSP70, TGF- $\beta$ and IGF-1 protein levels.

\section{Discussion}

Tendon injury often results in the formation of tendon adhesion, causing the deterioration of the range of motion of the joint (21). Patellar tendon adhesion substantially influences patellofemoral and tibiofemoral biomechanics and exhibits a close association with tendon shortening, changes of patellofemoral contact and tibiofemoral contact and knee arthrofibrosis (22). HSP-72 is upregulated in response to heat and mechanical stress, and this response is mediated by human fibroblasts and involves the nuclear translocation of HSP-72 (23). In the present study, the effect of HSP70 

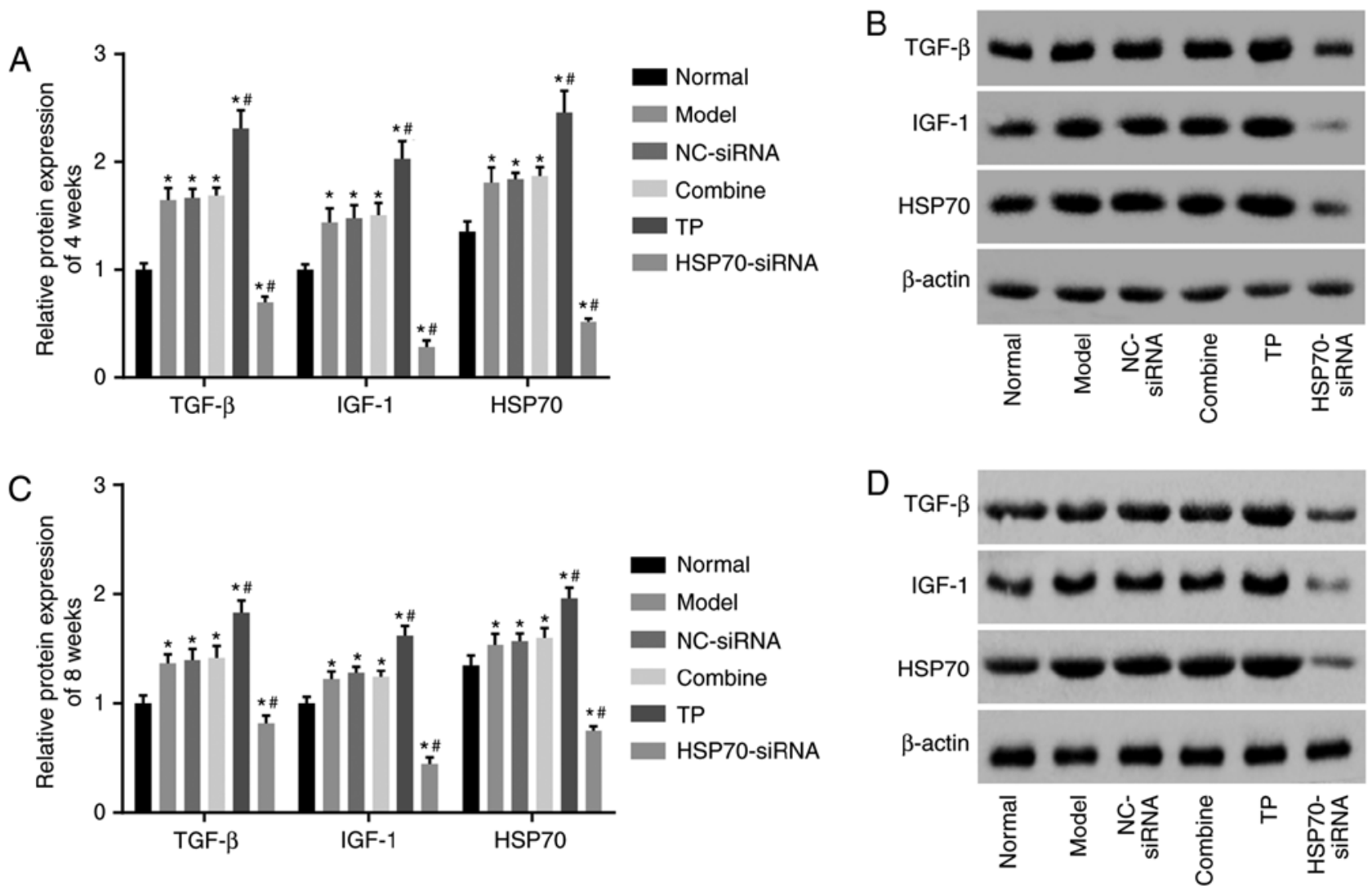

Figure 6. Western blot analysis demonstrated that TP increased HSP70, TGF- $\beta$ and IGF-1 protein levels. There were 10 rats in each group, with 5 rats observed 4 weeks following surgery and 5 rats observed 8 weeks following surgery. (A) Quantification analysis of HSP70, TGF- $\beta$ and IGF-1 protein levels in each group at the 4th week following surgery. (B) Protein bands of HSP70, TGF- $\beta$ and IGF-1 4 weeks following surgery in each group. (C) Quantification analysis of HSP70, TGF- $\beta$ and IGF-1 protein levels in each group at the 8th week following surgery. (D) Protein bands of HSP70, TGF- $\beta$ and IGF-1 8 weeks following surgery in each group. Data were analyzed by repeat measures analysis of variance. ${ }^{*} \mathrm{P}<0.05$ vs. the normal group; ${ }^{\#} \mathrm{P}<0.05 \mathrm{vs}$. the model group. NC, negative control; TP, thermal pretreatment; HSP, heat shock protein; siRNA, small interfering RNA; TGF- $\beta$, tumor growth factor $\beta$; IGF-1, insulin-like growth factor 1.

expression following the completion of TP on tendon healing and adhesion was investigated. The results revealed that TP increased HSP70 expression while reducing traumatic inflammation and postoperative adhesion in the tendon, thereby improving the tendon healing process in rats.

One of the major conclusions is that tendon adhesion is associated with HSP70. When HSP70 expression was reduced, tendon adhesion increased. HSPs are ubiquitous proteins that serve critical functions in the response to biotic and abiotic stresses (24). A previous study by Peterson et al (25) demonstrated that HSPs promote epithelial wound healing in various tissues, whereas reduced HSP70 expression may potentially prolong the healing process in the pathologic cornea of animals. Certain HSP70s, including molecular chaperones, serve a pivotal function in various biological processes, including the regulation of protein folding, interactions between proteins and translocation and degradation across membranes (26). A previous study indicated that HSP70 suppresses heat-induced apoptosis via inactivation of BCL2 associated $\mathrm{X}$, apoptosis regulator; thus, preventing the release of pro-apoptotic factors from the mitochondrial intermembrane space (27). HSP also serves a cytoprotective function in stressed muscle (28). Furthermore, a study consistent with the results of the present study demonstrated that the HSP70 expression level in chondrocytes exhibited a close association with the histological severity in osteoarthritis (29). In addition,
HSP70 overexpression promotes the metabolic activity of chondrocytes while protecting the chondrocytes from multiple stresses (30).

In addition, the present study provided evidence that TP increased HSP70 expression while reducing traumatic inflammation and tendon adhesion following surgery. TP alters tissue stiffness through the regulation of collagen composition, altering tissue susceptibility to histotripsy (31). As previously reported, TP is associated with the induction of HSP70 protein synthesis, and this mechanism may mitigate tissue damage in lung fibrosis (32). Li et al (33) also identified that appropriate TP may substantially increase HSP70 expression levels, reduce serum aspartate aminotransferase and alanine aminotransferase levels, decrease liver injury and promote liver repair following carbon tetrachloride-induced liver injury. In contrast, HSPs additionally influence cell protection, inflammation and immune system function (34). In addition, as an anti-apoptotic chaperone and through its chaperone activity, HSP70 tightly regulates inflammatory responses via the nuclear factor $-\kappa \beta$ signaling pathway, which is a critical pathway in mediating inflammatory responses (35). A previous study additionally demonstrated that TP reduced tendon adhesion via upregulating HSP72 expression (36). Furthermore, HSPs serve a substantial role in dealing with environmental stresses, including heat stress, whereas TP increases the mRNA levels of rhopalosiphum padi HSPs (37). 
To conclude, the results obtained in the present study also demonstrated that TP promoted tendon healing via its increase in tensile strength and tendon sliding distance. Heat stress contributed to satellite cell proliferation and protein synthesis in the repair of injured skeletal muscle. Thus, the recovery of injured skeletal muscle may benefit from heating (38). HSP70, TGF- $\beta$ and IGF-1 protein levels were found to be increased in the TP group but decreased in the HSP70-siRNA group in the present study. TGF- $\beta$ exerts a positive effect on tendon healing through the knockout of TGF- $\beta$ inducible early gene in the absence of the Smad pathway (39). One study demonstrated that increased TGF- $\beta$ expression of bone marrow-derived mesenchymal stem cells facilitates tendon healing via modulating the TGF- $\beta /$ mitogen activated protein kinase signaling pathway (40). Furthermore, TGF- $\beta 1$ and IGF-1 served a notable role in tendon cell proliferation and tendon tissue regeneration, and these processes were beneficial in the tendon healing process by regulating tenocyte proliferation and collagen metabolism (41). Another study also demonstrated that IGF-1, as an exogenous modulator, served a critical role in the promotion of tendon healing without promoting tendon adhesion given that it induces cell proliferation and collagen production and functions as strong angiogenetic factor (42). Therefore, the present study hypothesized that the increased expression of TGF- $\beta$ and IGF-1 induced by TP promotes tendon healing.

In conclusion, increased HSP70 expression following $\mathrm{TP}$ contributes to tendon healing in rats through attenuating traumatic inflammation and reducing postoperative tendon adhesion. Therefore, the present study provided an experimental baseline for the prevention of tendon adhesion in the clinic. However, due to the lack of a guaranteed prevention/treatment method, further studies are mandatory to obtain a more comprehensive understanding of the specific mechanisms involved with increased HSP70 expression following TP and how it benefits tendon healing.

\section{Acknowledgements}

Not applicable.

\section{Funding}

No funding was received.

\section{Availability of data and materials}

The datasets used and/or analyzed during the present study are available from the corresponding author on reasonable request.

\section{Authors' contributions}

XMT and JD wrote the paper, and conceived and designed the experiments; HLS analyzed the data; XMT and HLS collected and provided the samples for this study. JD wrote and revised the paper. All the authors approved the final version of the paper.

\section{Ethics approval and consent to participate}

The animal experiments were conducted in accordance with the Guide for the Care and Use of Laboratory Animals as promulgated by the National Institutes of Health. Ethical approval was obtained from the ethics committee of The Affiliated Huai'an No. 1 People's Hospital of Nanjing Medical University (Huai'an, China).

\section{Patient consent for publication}

Not applicable.

\section{Competing interests}

The authors declare that they have no competing interests.

\section{References}

1. Longo UG, Lamberti A, Maffulli N and Denaro V: Tissue engineered biological augmentation for tendon healing: A systematic review. Br Med Bull 98: 31-59, 2011

2. Bauge C, Leclercq S, Conrozier T and Boumediene $\mathrm{K}$ : TOL19-001 reduces inflammation and MMP expression in monolayer cultures of tendon cells. BMC Complement Altern Med 15: 217, 2015.

3. Wu PT, Jou IM, Kuo LC and Su FC: Intratendinous injection of hyaluronate induces acute inflammation: A possible detrimental effect. PLoS One 11: e0155424, 2016.

4. Geary MB, Orner CA, Bawany F, Awad HA, Hammert WC, O'Keefe RJ and Loiselle AE: Systemic ep4 inhibition increases adhesion formation in a murine model of flexor tendon repair. PLoS One 10: e136351, 2015.

5. Thomopoulos S, Das R, Sakiyama-Elbert S, Silva MJ, Charlton N and Gelberman RH: bFGF and PDGF-BB for tendon repair: Controlled release and biologic activity by tendon fibroblasts in vitro. Ann Biomed Eng 38: 225-234, 2010.

6. Dabak TK, Sertkaya O, Acar N, Donmez BO and Ustunel I: The effect of phospholipids (surfactant) on adhesion and biomechanical properties of tendon: A rat achilles tendon repair model. Biomed Res Int 2015: 689314, 2015.

7. Ackermann PW, Franklin SL, Dean BJ, Carr AJ, Salo PT and Hart DA: Neuronal pathways in tendon healing and tendinopathy-update. Front Biosci (Landmark Ed) 19: 1251-1278, 2014.

8. Mulhall KJ, McLaughlin R, Kay E, Kiely P, Bouchier-Hayes D and Murray P: Thermal preconditioning prevents peritendinous adhesions and inflammation. Clin Orthop Relat Res 405: 258-266, 2002.

9. Zhang L, Fok JH and Davies FE: Heat shock proteins in multiple myeloma. Oncotarget 5: 1132-1148, 2014.

10. Afzal E, Ebrahimi M, Najafi SM, Daryadel A and Baharvand H: Potential role of heat shock proteins in neural differentiation of murine embryonal carcinoma stem cells (P19). Cell Biol Int 35: 713-720, 2011.

11. Rauch JN and Gestwicki JE: Binding of human nucleotide exchange factors to heat shock protein 70 (Hsp70) generates functionally distinct complexes in vitro. J Biol Chem 289: 1402-1414, 2014.

12. Vazzana M, Siragusa T, Arizza V, Buscaino G and Celi M: Cellular responses and HSP70 expression during wound healing in Holothuria tubulosa (Gmelin, 1788). Fish Shellfish Immunol 42: 306-315, 2015.

13. Lancaster GI and Febbraio MA: Exosome-dependent trafficking of HSP70: A novel secretory pathway for cellular stress proteins. J Biol Chem 280: 23349-23355, 2005.

14. Mushtaq S, Naqvi ZA, Siddiqui AA and Ahmed N: Albumin precursor and Hsp70 modulate corneal wound healing in an organ culture model. Acta Histochem 113: 36-42, 2011.

15. Guide for the Care and Use of Laboratory Animals. Washington (DC), National Academies Press (US), 2011, vol. $10.17226 / 12910 \mathrm{p} . \wedge$ pp.

16. Cury DP, Schäfer BT, de Almeida SRY, Righetti MMDS and Watanabe IS: Application of a purified protein from natural latex and the influence of suture type on achilles tendon repair in rats. Am J Sports Med 47: 901-914, 2019.

17. Han Q, Li L, Liang H, Li Y, Xie J and Wang Z: Downregulation of lncRNA X inactive specific transcript (XIST) suppresses cell proliferation and enhances radiosensitivity by upregulating mir-29c in nasopharyngeal carcinoma cells. Med Sci Monit 23: 4798-4807, 2017. 
18. Kim KM, Wagle S, Moon YJ, Wang SI, Park BH, Jang KY and Kim JR: Interferon $\beta$ protects against avascular osteonecrosis through interleukin 6 inhibition and silent information regulator transcript-1 upregulation. Oncotarget 9: 3562-3575, 2017.

19. Dumas P, Morin MD, Boquel S, Moffat CE and Morin PJ: Expression status of heat shock proteins in response to cold, heat, or insecticide exposure in the Colorado potato beetle Leptinotarsa decemlineata. Cell Stress Chaperones 2019 (Epub ahead of print).

20. Song H, Xu Y, Shi L, Xu T, Fan R, Cao M, Xu W and Song J: LncRNA THOR increases the stemness of gastric cancer cells via enhancing SOX9 mRNA stability. Biomed Pharmacother 108: 338-346, 2018

21. Loiselle AE, Bragdon GA, Jacobson JA, Hasslund S, Cortes ZE, Schwarz EM, Mitten DJ, Awad HA and O'Keefe RJ: Remodeling of murine intrasynovial tendon adhesions following injury: MMP and neotendon gene expression. J Orthop Res 27: 833-840, 2009

22. Fernandez JW, Akbarshahi M, Crossley KM, Shelburne KB and Pandy MG: Model predictions of increased knee joint loading in regions of thinner articular cartilage after patellar tendon adhesion. J Orthop Res 29: 1168-1177, 2011.

23. Jagodzinski M, Hankemeier S, van Griensven M, Bosch U, Krettek $\mathrm{C}$ and Zeichen J: Influence of cyclic mechanical strain and heat of human tendon fibroblasts on HSP-72. Eur J Appl Physiol 96: 249-256, 2006

24. Liu J, Wang R, Liu W, Zhang H, Guo Y and Wen R: Genome-wide characterization of heat-shock protein 70 s from chenopodium quinoa and expression analyses of cqhsp70s in response to drought stress. Genes (Basel) 9: E35, 2018.

25. Peterson C, Driskell E, Wilkie D, Premanandan C and Hamor R Heat-shock protein 70 expression in the equine cornea. Vet Ophthalmol 20: 344-348, 2017.

26. Kampinga HH and Craig EA: The HSP70 chaperone machinery: $\mathrm{J}$ proteins as drivers of functional specificity. Nat Rev Mol Cell Biol 11: 579-592, 2010.

27. Stankiewicz AR, Lachapelle G, Foo CP, Radicioni SM and Mosser DD: Hsp70 inhibits heat-induced apoptosis upstream of mitochondria by preventing Bax translocation. J Biol Chem 280 38729-38739, 2005.

28. Inoue T, Suzuki S, Hagiwara R, Iwata M, Banno Y and Okita M Effects of passive stretching on muscle injury and HSP expression during recovery after immobilization in rats. Pathobiology 76 : 253-259, 2009

29. Son YO, Kim HE, Choi WS, Chun $\mathrm{CH}$ and Chun JS: RNA-binding protein ZFP36L1 regulates osteoarthritis by modulating members of the heat shock protein 70 family. Nat Commun 10: 77, 2019.

30. Tonomura H, Takahashi KA, Mazda O, Arai Y, Shin-Ya M, Inoue A, Honjo K, Hojo T, Imanishi J and Kubo T: Effects of heat stimulation via microwave applicator on cartilage matrix gene and HSP70 expression in the rabbit knee joint. J Orthop Res 26 : 34-41, 2008.

31. Vlaisavljevich E, Xu Z, Arvidson A, Jin L, Roberts W and Cain C: Effects of thermal preconditioning on tissue susceptibility to histotripsy. Ultrasound Med Biol 41: 2938-2954, 2015.
32. Hagiwara S, Iwasaka $H$, Matsumoto $S$, Noguchi $T$ and Yoshioka H: Association between heat stress protein 70 induction and decreased pulmonary fibrosis in an animal model of acute lung injury. Lung 185: 287-293, 2007.

33. Li SQ, Wang DM, Shu YJ, Wan XD, Xu ZS and Li EZ: Proper heat shock pretreatment reduces acute liver injury induced by carbon tetrachloride and accelerates liver repair in mice. J Toxicol Pathol 26: 365-373, 2013.

34. Kasperska-Zajac A, Damasiewicz-Bodzek A, Bieniek K, Skrzypulec-Frankel A, Tyrpien-Golder K and Grzanka A: Elevated circulating heat shock protein 70 and its antibody concentrations in chronic spontaneous urticaria. Int $\mathrm{J}$ Immunopathol Pharmacol 31: 394632017750440, 2018.

35. Sevin M, Girodon F, Garrido C and de Thonel A: HSP90 and HSP70: Implication in inflammation processes and therapeutic approaches for myeloproliferative neoplasms. Mediators Inflamm 2015: 970242, 2015

36. Tan Y, Wu QF, Wu Q, Tan XT, Chen LB and Wang X: Thermal preconditioning may prevent tendon adhesion by up-regulating hsp72 in rats. Cell Physiol Biochem 42: 1623-1634, 2017.

37. Li Y, Zhao Q, Duan X, Song C and Chen M: Transcription of four Rhopalosiphum padi (L.) heat shock protein genes and their responses to heat stress and insecticide exposure. Comp Biochem Physiol A Mol Integr Physiol 205: 48-57, 2017.

38. Kojima A, Goto K, Morioka S, Naito T, Akema T, Fujiya H, Sugiura T, Ohira Y, Beppu M, Aoki H and Yoshioka T: Heat stress facilitates the regeneration of injured skeletal muscle in rats. J Orthop Sci 12: 74-82, 2007.

39. Tsubone T, Moran SL, Subramaniam M, Amadio PC, Spelsberg TC and An KN: Effect of TGF-beta inducible early gene deficiency on flexor tendon healing. J Orthop Res 24: 569-575, 2006.

40. Wang R, Xu B and Xu HG: Up-regulation of tgf-beta promotes tendon-to-bone healing after anterior cruciate ligament reconstruction using bone marrow-derived mesenchymal stem cells through the tgf-beta/mapk signaling pathway in a new zealand white rabbit model. Cell Physiol Biochem 41: 213-226, 2017.

41. Chen YJ, Wang CJ, Yang KD, Kuo YR, Huang HC, Huang YT, Sun YC and Wang FS: Extracorporeal shock waves promote healing of collagenase-induced achilles tendinitis and increase TGF-beta1 and IGF-I expression. J Orthop Res 22: 854-861, 2004.

42. Berglund ME, Hart DA, Reno C and Wiig M: Growth factor and protease expression during different phases of healing after rabbit deep flexor tendon repair. J Orthop Res 29: 886-892, 2011.

This work is licensed under a Creative Commons Attribution-NonCommercial-NoDerivatives 4.0 International (CC BY-NC-ND 4.0) License. 